\title{
Improved Kalman Filter Based Inverter Control for Reduction of Low Order Current Harmonics due to Isolation Transformers in Renewable Energy Sources
}

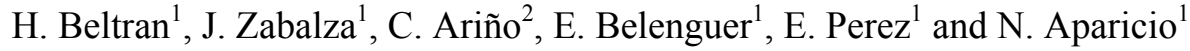 \\ ${ }^{1}$ Electrical Engineering Area \\ Department of Industrial Systems Engineering and Design \\ ESTCE, Universitat Jaume I de Castelló \\ Phone/Fax number: +34964 728178, e-mail: hbeltran@esid.uji.es, jzabalza@invate.es
}

\author{
${ }^{2}$ Systems Engineering and Automatic Area \\ Department of Industrial Systems Engineering and Design \\ ESTCE, Universitat Jaume I de Castelló \\ Phone/Fax number: +34 964 728183, e-mail: arino@esid.uii.es
}

\begin{abstract}
This paper is focused on the analysis of a Kalman filter performance when introduced in the current control loop of a distributed generation connection inverter. The idea is to use this kind of filter to reduce the harmonic content of the currents injected in the point of common coupling. The study has been performed by means of simulation using MatlabSimulink and clearly demonstrates that this kind of control system can improve the response produced by the inverters. The elimination of certain well-known noisy harmonics introduced by an element such as the isolation low voltage transformer has been accomplished.
\end{abstract}

\section{Key words}

Distributed generation, harmonic compensation, Kalman filters, renewable energy efficiency.

\section{Introduction}

One of the new problems encountered in the Distributed Generation (DG) electric philosophy is the large introduction of harmonics produced by the electric components which connect the renewable Distributed Resources (DR) with the Electric Power System (EPS). Both inverters and connection transformers create new current harmonics which interfere with the normal performance of the low voltage distribution networks. The nature of these harmonics is quite different being mainly high frequency current harmonics, in the range of $1 \mathrm{kHZ}$ to $30 \mathrm{kHZ}$, for the case of the inverters (depending on the switching frequency of the converter) and harmonics which comprehend mainly low frequency range (highlighting the $5^{\text {th }}$ and $7^{\text {th }}$ harmonic), for the case of the transformers. These low frequency harmonics are typically characterized by the magnetic core of the transformer and correspond to its magnetization currents [1].

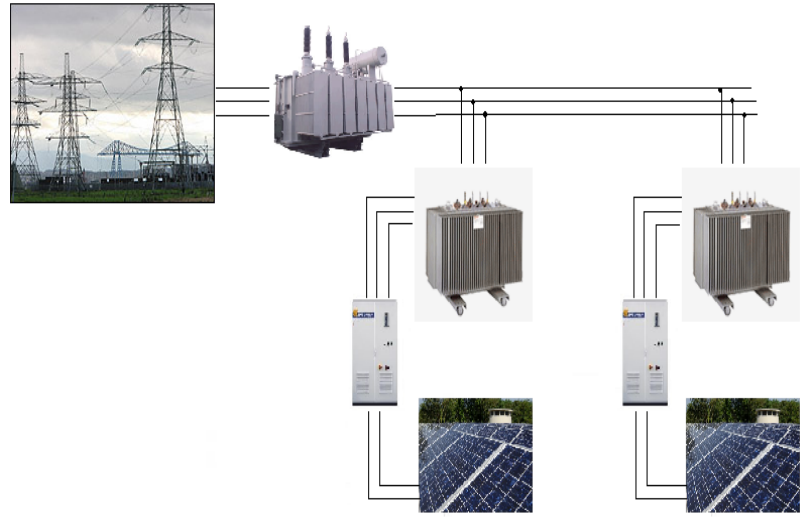

Fig. 1. PV distributed system connected to the EPS

It is important to point out that most of the photovoltaic $(\mathrm{PV})$ inverters installed to the moment and many of those still commercially available in the market just generate the reference currents to inject into the EPS by detecting the zero crossing point of the voltage at the point of common coupling (PCC) and following a sinusoidal wave from there on. In that sense, they cannot take into account the existence of harmonics since they are not internally programmed. Moreover, the control is only refreshed every $20 \mathrm{~ms}$ with each zero crossing.

The interest of the work introduced on this paper is the reduction of the harmonic content included in the current injected by these PV inverters. The idea is not only to make them generate a sinusoidal wave in phase with the voltage at the PCC, but also some harmonics superposed on it which would compensate the harmonics consumed by the isolation or connection transformer. This reduction is obtained by introducing a Kalman filter in the current control system of the different converters. Kalman filters 
have been previously used in power electronics control for applications such as the tracking of the fundamental voltage wave at the point of common connection (PCC) of the different DGs [2].

In the field of the electrical engineering, Kalman filters have been used for long in applications such as the electric motors characteristic parameters measurement $[3,4]$ or the harmonics detection [5]. The use now of Kalman filters in the current control loop can be seen as a new way to cancel not only the current harmonics demanded by the isolation or connection transformer but also to remove any undesired component of the current, if required, or even modify the shape of the injected current to accomplish certain criteria (reactive or unbalance).

As it has been introduced in the abstract of the document the main objective of this paper is to check the viability and performance of introducing a Kalman filter in the current control loop of the connection inverters used by the low voltage distributed generators. The goal is to study what the system response is when the filter is implemented to periodical harmonics, which remain in the time as those generated mainly by the isolation transformer. Another objective of the paper was to compare the performance of the proposed harmonic cancellation method with other published methods and already implemented, this work has been postponed to future publications.

\section{Kalman filters}

The Kalman filter [6] is an efficient recursive filter that estimates the state of a dynamic system from a series of noisy measurements. Generally, it tries to estimate the state of a controlled process which follows a state equation where the noise of the system has been added:

$$
\dot{x}(t)=A \cdot x(t)+B \cdot u(t)+w(t)
$$

Where $x(t)$ are the states and $u(t)$ the inputs. For that estimation, it uses as well the output signals equation where the noise corresponding to the measurement has been added too.

$$
y(t)=C \cdot x(t)+v(t)
$$

In these equations, $w(t)$ and $v(t)$ are respectively the noises of the system and that related to the measurement. Those noises are supposed not to be correlated in time and presenting normal or Gaussian probabilistic distributions. Hence, they are independent.

$$
\begin{aligned}
& p(w) \sim N(0, Q) \\
& p(v) \sim N(0, R)
\end{aligned}
$$

The state estimate $\hat{x}(t)$ that minimizes the steadystate error covariance

$$
P=\lim _{t \rightarrow \infty} E\left([x-\hat{x}][x-\hat{x}]^{T}\right)
$$

Is defined as

$$
\dot{\hat{x}}(t)=A \cdot \hat{x}(t)+B \cdot u(t)+L(y(t)-C \hat{x}(t))
$$

In order to simplify, the matrix $L$ in (5) is considered to be constant in time.

It is important to remark Kalman filters have been widely spread in the industry [7]. They are used in a wide range of applications such different from each other as robotics control, spacecraft attitude estimation [8] or even depth estimation from images [9] and, in general, any kind of multivariable control where no every state is accessible.

As previously introduced, in the field of the electrical engineering, Kalman filters have been used for long in applications such as the electric motors characteristic parameters measurement or the current harmonics detection. And although Kalman filters have been previously used in power electronics control for applications such as the tracking of the fundamental voltage wave at the point of common connection (PCC) of the different DGs, its use now in the current control loop can be seen as a new way to cancel the current harmonics, demanded by the isolation or connection transformer.

The current signal $y(t)$ with $n$ harmonics is modelled as

$$
y=x_{1}+x_{3}+\ldots+x_{2 n-1}
$$

Where

$$
x_{2 i-1}=A_{i} \sin \left(\omega_{i} t+\varphi_{i}\right) i=1 \ldots n
$$

This model can be represented as the following state space system without inputs.

$$
\begin{gathered}
{\left[\begin{array}{c}
\dot{x}_{1} \\
\dot{x}_{2} \\
\vdots \\
\dot{x}_{2 n-1} \\
\dot{x}_{2 n}
\end{array}\right]=\left[\begin{array}{ccccc}
0 & 1 & \ldots & 0 & 0 \\
-\omega_{1}^{2} & 0 & \ldots & 0 & 0 \\
\vdots & \vdots & \ddots & \vdots & \vdots \\
0 & 0 & \ldots & 0 & 1 \\
0 & 0 & \ldots & -\omega_{n}^{2} & 0
\end{array}\right] x} \\
y=\left[\begin{array}{lllll}
1 & 0 & \ldots & 1 & 0
\end{array}\right] x
\end{gathered}
$$

As the model (8), (9) has the structure described in (1), (2), the Kalman filter can be applied.

To test the validity of the model implemented in our system, a simulation is carried out where a signal which could perfectly be the actual current injected by the DG at the EPS, is feeded to the Kalman filter. This current comprehends the fundamental and two harmonics, $5^{\text {th }}$ and $7^{\text {th }}$, with small values. 


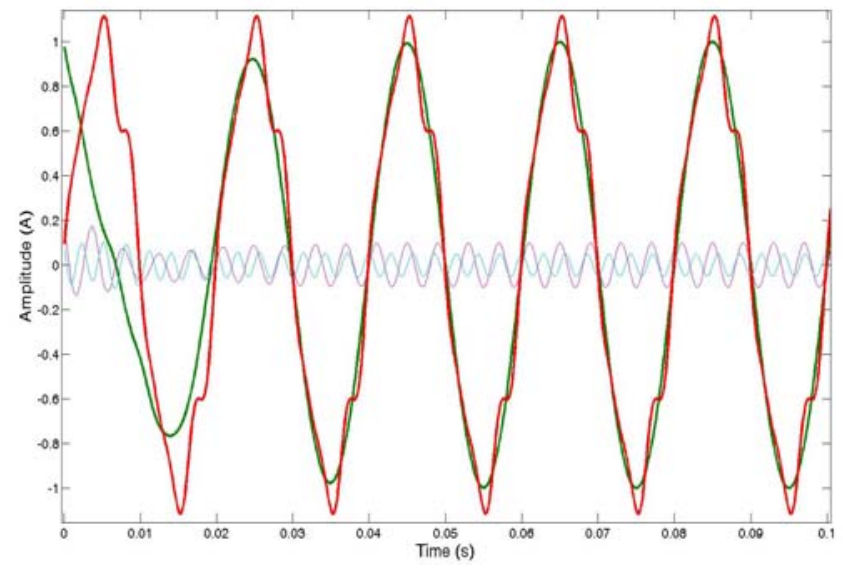

Fig. 2. Separation of harmonics in a Kalman Filter.

Figure 2 represents the output of our Kalman filter where the filtering of the different signals, representing the fundamental and two low frequency harmonics is stated. The red line in the figure represents the input and the green, blue and lilac lines represent the estimations of the fundamental, $5^{\text {th }}$ and $7^{\text {th }}$ harmonics. The detection and separation of the different harmonics existing in the initial polluted signal can be clearly observed.

With the introduction of the Kalman filter, the 3 waves are outputted allowing the introduction to the inverter of new references and not only that of the fundamental. Since we know now what the harmonics we are producing are, we can decide to generate the harmonics opposed in phase to those existing, in order to compensate and reduce in this way the THD at the PCC.

This harmonic cancellation system is quick, robust and much easier to implement in the digital devices in charge of the inverters' control than other methods not based on matrix calculation. Hence, the proposed control system can contribute to enhance the performance of the inverters in an easy way and improve the viability of the DG system paradigm as well as the functioning of isolated microgrids.

\section{Description of the system implemented}

In order to proceed with the analysis of a Kalman filter performance when introduced in the current control loop of a distributed generation connection inverter, we have developed a digital Matlab/Simulink model. The main structure of this model can be appreciated in figure 3 .

The system has been simplified, referred to a whole low voltage distribution system with different distributed generators, in order to focus the study in the efficiency of the Kalman filter.

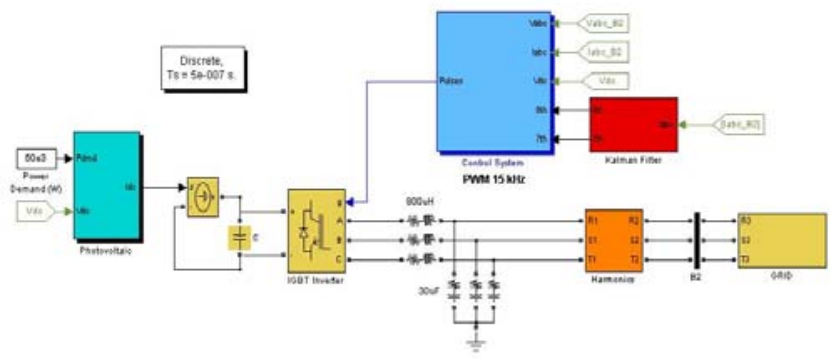

Fig. 3. Simulated DG system Matlab model.

In this way, the system under simulation is composed just by one distributed generator, a photovoltaic system, which injects current into the EPS (block on the top right of the figure called "Grid"). The injection of energy from the power inverter is done through its corresponding isolation transformer which is, as it has been introduced, the main source of production of low frequency harmonics (square orange block next to the grid called "Harmonics").

The PV connection inverter has been modelled by means of a full IGBT bridge converter with its corresponding dc bus and the passive filter on the ac side. The IGBTs are switched with a frequency of $15 \mathrm{kHZ}$ and the step of simulation is $0.5 \mu \mathrm{s}$. Together with the PV panels the power conversion stage can be observed in figure 3 on the left hand side. On the top of this figure, we have to point out the blocks corresponding to the measurements and control of the system, highlighting the Kalman filter in red colour. The Kalman filter, which is based on state equations, performs the separation of the fundamental component, the $5^{\text {th }}$ and the $7^{\text {th }}$ harmonics, all of them from the measured grid currents. Once we have these three signals clearly discerned, we can apply specific dq rotating frame controls for each harmonic, which complement the main one, working at the corresponding specific frequency. And these specific controls will eliminate the harmonics if we adjust their $\mathrm{d}-\mathrm{q}$ reference components to zero in our control.

Further description on the basis of the control system can be achieved with the help of figure 4 . As can be observed in the figure, we use 3 different rotating frames. They are a $50 \mathrm{~Hz}$ dq-frame control for the currents measured in the grid, and other two extra dq-frame controls, at 250 and $350 \mathrm{~Hz}$, for the $5^{\text {th }}$ and $7^{\text {th }}$ harmonic control respectively.

While for the harmonic current regulators, the reference "d" and "q" values are equal to zero (elimination of the harmonics), for the main dq-frame control, which turns at $50 \mathrm{~Hz}$, we will impose a "d" reference component (originated in a previous PI controller) that will regulate the voltage level at the dc bus of the convertes, and a " $q$ " reference component fixed to zero so the system can inject currents in the same phase angle that voltages. 


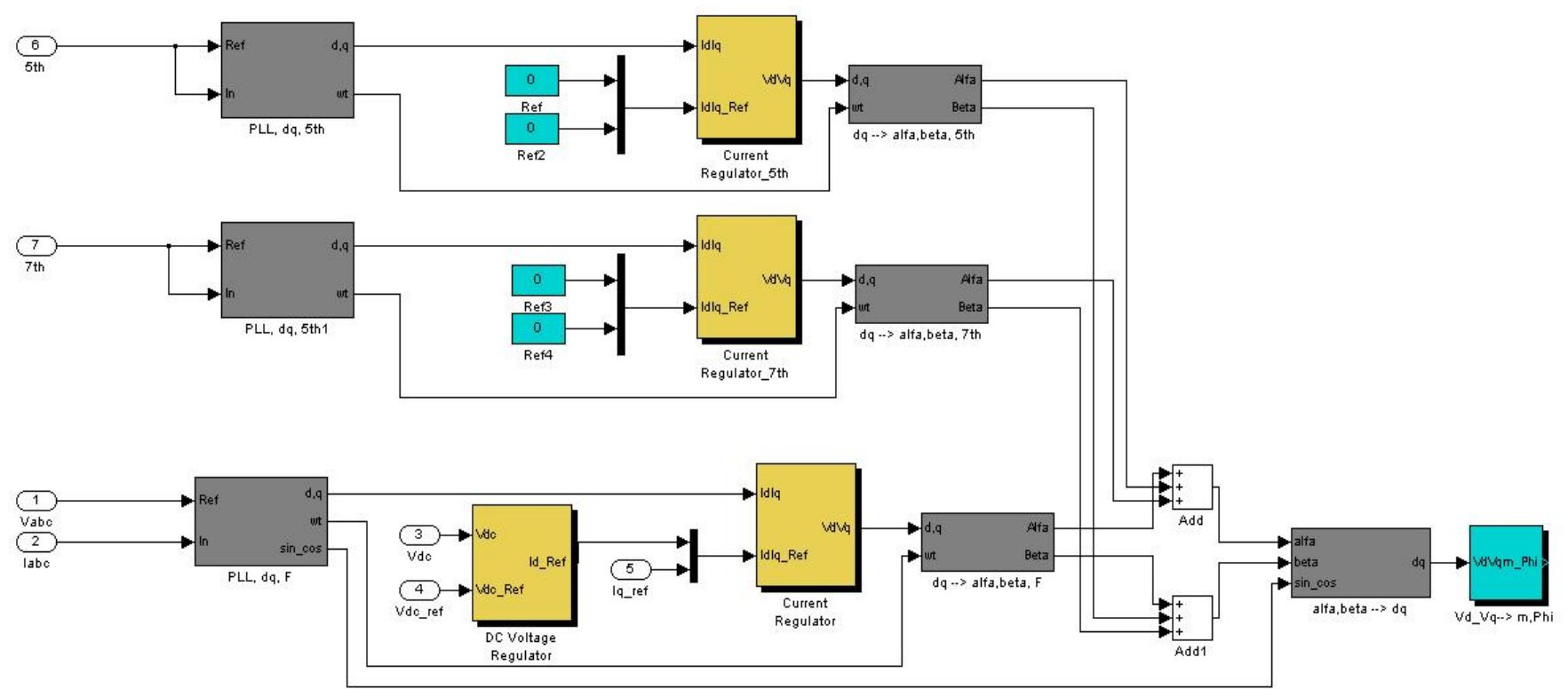

Fig. 4. Current reference generation control system.

In order to perform properly with the dq transformations, we force all the inputs (measured grid currents, $5^{\text {th }}$ and $7^{\text {th }}$ harmonic proceeding from the filter) go through a specific Phase Locked Loop (PLL), obtaining the representative dq components for each input and located on their rotating dq axis. Once these components are known, we apply PI controllers to each of them comparing with the reference values and obtaining output values for $d$ and q. Since each of the inputs has its own PLL working at a different frequency, they have different rotating $\mathrm{dq}$ axes. Hence, we have to transform the dq output components to their corresponding alpha-beta components. Then, once in the alpha-beta reference system (fix axis), we can add all alpha components and all beta components, reaching just one vector, with alphabeta components which represents the global system response. Finally, we transform this vector once again to the dq rotating frame at fundamental frequency $(50 \mathrm{~Hz})$, obtaining in this way the triggers for the inverter.

The reference pulsed signals obtained from the control system and introduced to the power converter are generated in phase with the voltage at the PCC, thanks to the PLL information which is introduced in that final conversion to the dq rotating frame. These reference signals will therefore not produce reactive power and neither $5^{\text {th }}$ nor the $7^{\text {th }}$ harmonic component.

\section{Results obtained}

With the model introduced in the previous section, we have simulated the existence of quite important harmonic levels in the current injected by the PV inverter into the EPS. As can be appreciated in the left column of table I, the overall THD of our model previous to the Kalman filter introduction is $15 \%$. This is a very high value which we have taken as a very bad situation in order to study the capacity of reduction of our filter.
Since magnetization currents of the transformer are approximately constant once it is connected, this level of distortion could be easily reached in a PV installation along several hours per day. Apart from the central daylight hours, when the power injected is similar to the rated one of the equipment, the rest of day and specially the daybreak hours and those of the sunset, the current injected will not be very higher than that consumed by the inverter. This may cause having a very high THD degree. And, as it has been pointed out before in the paper, this is a very typical situation when the inverter is programmed to inject simply a sinusoidal current wave in phase with the voltage after detecting its zero crossing.

The appearance of the current, seen from the point of view of the low voltage distribution network where the $\mathrm{PV}$ installation is connected is that of the figure 5. The distortion introduced on the fundamental wave by the $5^{\text {th }}$ and the $7^{\text {th }}$ harmonics are clearly appreciated. With our model and the Kalman filter implemented, these currents can be improved, compensating the harmonics till obtaining the currents waves depicted on figure 6 .

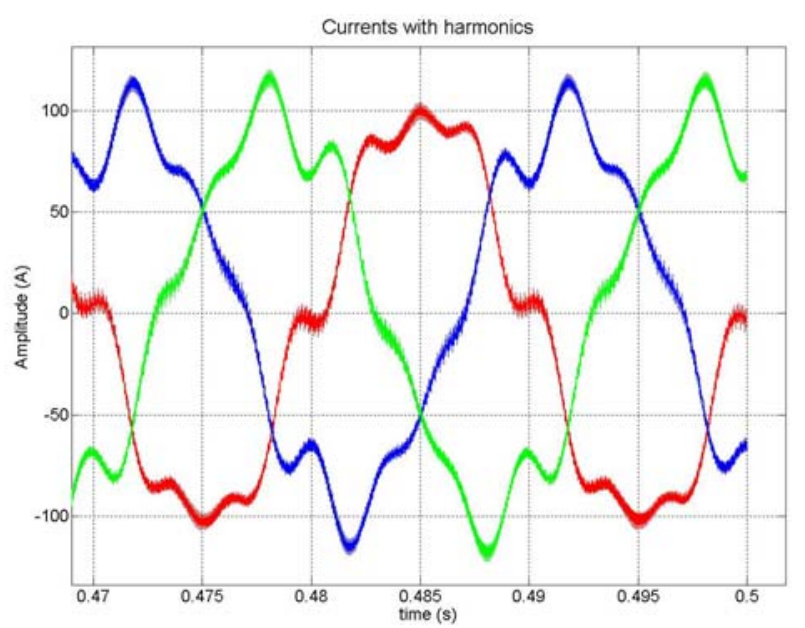

Fig.5. Currents injected to the EPS previous to filter activation 


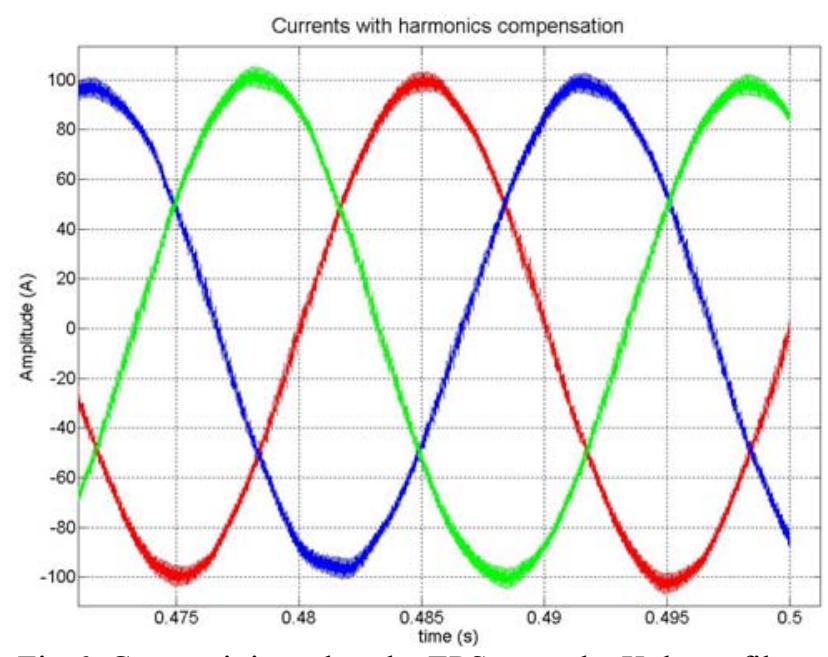

Fig.6. Current injected to the EPS once the Kalman filter is active

The improvement is evident and can be easily analyzed by comparing the values summarized in the two columns of Table I. The amplitude of the harmonic components is largely reduced and the value of the THD has drastically dropped to a $3 \%$. This new value is perfectly compatible with the limitations established by the Spanish laws to the connection of DGs to the EPS.

TABLE I. - Comparison of results when introducing the filter.

\begin{tabular}{|c|c|}
\hline WITHOUT FILTER & WITH KALMAN FILTER \\
\hline Total RMS $=72 \mathrm{~A}$ & Total $\mathrm{RMS}=71 \mathrm{~A}$ \\
\hline DC component $=0.1 \mathrm{~A}$ & DC component $=0.5 \mathrm{~A}$ \\
\hline RMS for $50 \mathrm{~Hz}=71 \mathrm{~A}$ & RMS for $50 \mathrm{~Hz}=71 \mathrm{~A}$ \\
\hline RMS harmonics $=11 \mathrm{~A}$ & RMS harmonics $=2 \mathrm{~A}$ \\
\hline Amplitude $50 \mathrm{~Hz}=100.5 \mathrm{~A}$ & Amplitude $50 \mathrm{~Hz}=100.2 \mathrm{~A}$ \\
\hline Amplitude $5 \mathrm{th}=10.5 \mathrm{~A}$ & Amplitude 5 th $=0.2 \mathrm{~A}$ \\
\hline Amplitude $7 \mathrm{th}=10.5 \mathrm{~A}$ & Amplitude $7 \mathrm{th}=0.04 \mathrm{~A}$ \\
\hline THD $=\mathbf{1 5} \%$ & THD $=\mathbf{3 \%}$ \\
\hline
\end{tabular}

In order to study the dynamics of the system, we have simulated the transition period corresponding to the moment when the filter starts functioning. This can be observed on figure 7 , where a stabilization time of around $0.2 \mathrm{~s}$ is appreciated. Similar simulations have been performed for the case of considering a change in the harmonic content of the current, studying in this way the time of response of the filter to the new situation and the quality of the new compensation. Both results seem quite satisfactory and encourage us to try to implement the filter in our lab test bench. Future experimental result should verify the validity of our solution.

\section{Conclusion}

The work introduced in this paper demonstrates by means of simulations, and using the software Matlab/Simulink with its library SimPowerSystems, the viability of reducing the overall THD in the current produced by the DRs connected in a low voltage distribution network.

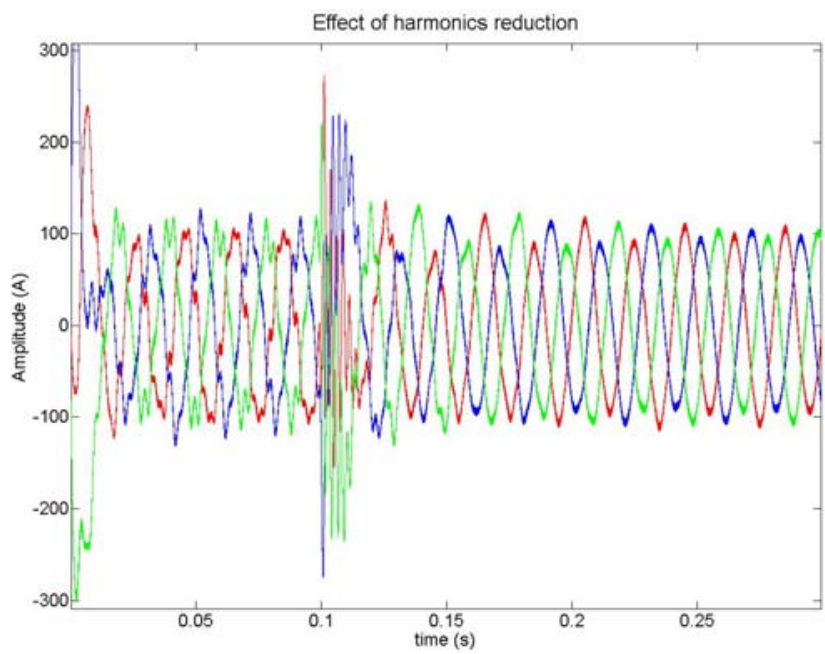

Fig.7. Evolution of the response after filter activation

The introduction and use of a Kalman filter, which feedbacks the current control loop complementing the information it has from the measurements, allows avoiding the appearance of distortions (in the form of periodic low frequency harmonics) in the injected current which are caused by the components located between the PCC and the exit of the inverter, where the current sensors are usually placed. The isolation transformer is the electric element existing there, with non-perfect linear response which may introduce these undesired low frequency harmonics. By changing the position of the current sensors, the feedback can encounter the existence of the harmonics and, with the help of the Kalman filter, change the current reference of the inverter in order to cancel the overall response of the system.

Thanks to previous works where the validity of our simulation models having been checked, we can now affirm with the results obtained in this paper that commercial PV inverters could improve greatly their efficiency and THD generation just by introducing this kind of filters in the current control loop. This would be cheap, easy and would provide the inverter higher functioning characteristics. Our work now is focused on verifying these results in our lab test bench in order to obtain experimental certification of the proposal.

\section{Acknowledgement}

This work has been supported by the IMPIVA (Institut de de la mitjana i petita industria de la Generalitat Valenciana)

\section{References}

[1] MIT E.E. Staff, Magnetic Circuits and Transformers, MIT Press 1943.

[2] K. De Brabandere, B. Bolsens, J. Van den Keybus, A. Woyte, J. Driesen and R. Belmans, "A voltage and frequency droop control method for parallel inverters", in Proc. PESC 2004, pp. 2501-2507.

[3] Li-Cheng Zai, Christopher L. DeMarco, and Thomas A. Lipo, "An Extended Kalman Filter Approach to Rotor Time Constant Measurement in PWM Induction Motor Drives", IEEE Trans. On Industry 
Applications, Vol. 28, No. 1, pp. 96-104, January/ February 1992.

[4] R. Dhaouadi, N. Mohan and L. Norum, "Design and implementation of an extended Kalman filter for the state estimation of a permanent magnet synchronous motor," Power Electronics, IEEE Transactions on, vol. 6, pp. 491-497, 1991.

[5] Aurobinda Routray, Ashok Kumar Pradhan, and K. Prahallad Rao, "A Novel Kalman Filter for Frequency Estimation of Distorted Signals in Power Systems", IEEE Trans. On Instrumentation and Measurement, Vol. 51, No. 3, pp. 469-479, June 2002.

[6] Kalman, R.E. (1960). "A new approach to linear filtering and prediction problems". Journal of Basic Engineering 82 (1): 35-45.
[7] B. D. O. Anderson, J. B. Moore and M. Eslami, "Optimal Filtering," Systems, Man and Cybernetics, IEEE Transactions vol. 12, pp on,. 235-236, 1982.

[8] E. J. Leffens, F. L. Markley and M. D. Shuster. (1982, 1982). "Kalman filtering for spacecraft attitude estimation." Journal of Guidance, Control, and Dynamics vol 5, pp. 417-429.

[9] L. Matthies, T. Kanade and R. Szeliski, "Kalman filter-based algorithms for estimating depth from image sequences," International Journal of Computer Vision, vol. 3, pp. 209-238, 09/01. 1989. 